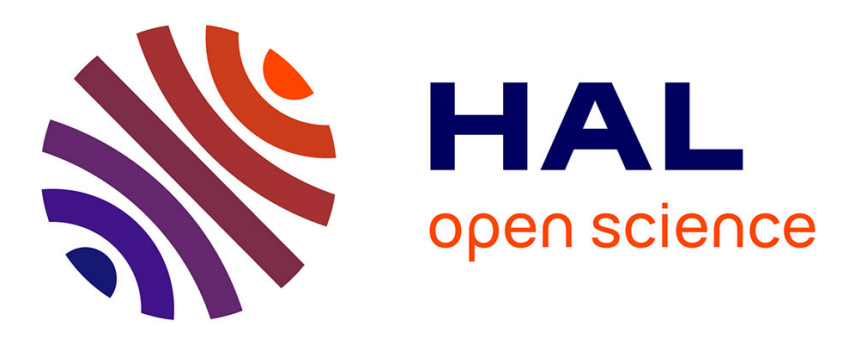

\title{
Clinical workflow for personalized foot pressure ulcer prevention
}

\author{
M. Bucki, V. Luboz, A. Perrier, E. Champion, B. Diot, N. Vuillerme, Yohan \\ Payan
}

\section{- To cite this version:}

M. Bucki, V. Luboz, A. Perrier, E. Champion, B. Diot, et al.. Clinical workflow for personalized foot pressure ulcer prevention. Medical Engineering \& Physics, 2016, 38 (9), pp.845-853. 10.1016/j.medengphy.2016.04.017 . hal-01403955

\section{HAL Id: hal-01403955 \\ https://hal.science/hal-01403955}

Submitted on 28 Nov 2016

HAL is a multi-disciplinary open access archive for the deposit and dissemination of scientific research documents, whether they are published or not. The documents may come from teaching and research institutions in France or abroad, or from public or private research centers.
L'archive ouverte pluridisciplinaire HAL, est destinée au dépôt et à la diffusion de documents scientifiques de niveau recherche, publiés ou non, émanant des établissements d'enseignement et de recherche français ou étrangers, des laboratoires publics ou privés. 


\section{Clinical workflow for personalized foot pressure ulcer prevention}

2

3

4

51 TexiSense, Montceau-les-Mines, France, \{antoine.perrier, vincent.luboz, marek.bucki,

6 eric.champion\}@texisense.com;

72 Univ. Grenoble Alpes \& CNRS, TIMC-IMAG, F-38000 Grenoble, France, \{antoine.perrier,

8 yohan.payan\}@imag.fr;

93 UJF-Grenoble1/AGEIS, Grenoble, F-38041, France, Nicolas.Vuillerme@ agim.eu

*Corresponding author. Tel.: +33 456520001 , fax: +33 456520055 .

E-mail address: Yohan.Payan@imag.fr.

\begin{abstract}
Foot pressure ulcers are a common complication of diabetes because of patient's lack of sensitivity due to neuropathy. Deep pressure ulcers appear internally when pressures applied on the foot create high internal strains nearby bony structures. Monitoring tissue strains in persons with diabetes is therefore important for an efficient prevention. We propose to use personalized biomechanical foot models to assess strains within the foot and to determine the risk of ulcer formation. Our workflow generates a foot model adapted to a patient's morphology by deforming an atlas model to conform it to the contours of segmented medical images of the patient's foot. Our biomechanical model is composed of rigid bodies for the bones, joined by ligaments and muscles, and a Finite Element mesh representing the soft tissues. Using our registration algorithm to conform three datasets, three new patient models were created. After applying a pressure load below these foot models, the Von Mises equivalent strains and "cluster volumes" (i.e. volumes of contiguous elements with strains above a given threshold) were measured within eight functionally meaningful foot regions. The results show the variability of both location and strain values among the three considered patients. This study also confirms that the anatomy of the foot has an influence on the risk of pressure ulcer.
\end{abstract}

Keywords: Foot pressure ulcer; Soft tissues; Patient-Specific; Finite element method.

\section{Introduction}

It has been estimated that a limb is lost every 30 seconds in the world due to diabetes. This trend is expected to be multiplied by four in the next 15 years with the pandemic evolution of diabetes 
(Shaw et al., 1997). In addition to causing pain and morbidity, foot lesions in diabetic patients have substantial direct and indirect economic consequences (Shearer et al., 2003)(Gordois et al., 2003). Diabetic foot ulcers result from multiple pathophysiological mechanisms, including neuropathy, peripheral vascular disease, high foot pressures, foot deformity, and diabetes severity (Telfer et al., 2014). Several studies (Mueller, 1992)(Loerakker et al., 2011) recognized at least three mechanisms leading to pressure ulcer: (1) ischemia caused by increased pressure duration even for low induced strains, (2) high internal tissue strains created by increased pressure magnitude, and/or (3) tissue fatigue caused by increased number of periodic pressure loads. Time and strain have an inversely proportional contribution to ulceration (Kosiak, 1959)(Loerakker et al., 2011)(Van Schie et al., 2006): high strains take a relatively short time (a few minutes) to cause ulceration whereas low strains induce lesion after a longer period (between two and four hours). Short and long term lesion inducing strain thresholds have been characterized by (Loerakker et al., 2011) in muscle tissues. The obtained values were around $50 \%$ of deformation for short term high strains and $20 \%$ of deformation for long term low strains. This study also showed that fat tissues have large strain variations (although not as large as muscle tissues) and they might suffer from pressure ulcer. The two strain thresholds aforementioned are therefore key values in pressure ulcer prevention.

Daily monitoring by the patient or clinical staff is the main tool to prevent foot pressure ulcers and results in an estimated reduction of foot ulcers and amputations from 50\% to 80\% (Boulton et al., 2005). Because early stages of ulceration are not always visible, both patient's and staff's vigilances tend to decrease over time. Unfortunately, in the case of diabetic patients, it is precisely when the first ulcers appear that serious complications develop, mainly because of the angiopathy, which severely limits healing.

It is consequently essential to introduce new monitoring tools to promote awareness and as a result, patient's autonomy in everyday life. Measuring pressure loads at the skin surface, all around the foot, and, if possible, estimating the corresponding internal strains could help preventing further ulceration and facilitate wound healing (Gefen, 2010). Measuring interface pressures can be performed with a pressure sensor such as the ones proposed by Novel (http://www.novel.de), Tekscan (http://www.tekscan.com), Vista Medical (http://www.pressuremapping.com) or Texisense (http://www.texisense.com), however several studies established that using pressure measurements at the skin interface is not sufficient to prevent foot pressure ulcers, especially the ones starting deep in the tissues and causing substantial subcutaneous damage underneath intact skin (Linder-Ganz et al., 2008)(Atlas et al., 2009)(Gefen, 2003). Indeed surface measurements do not provide enough information as to predict ulcer formation in a reliable way (Linder-Ganz et al., 2008). For example, with the same pressure map, a patient with a sharp calcaneus, or a thinner heel pad, could develop a pressure ulcer while another one, with a different morphology, might not. Pressure ulcer risk is consequently highly patient-related and integrates a number of factors such as bones' curvature (Luboz et al., 2015), or soft tissue thickness (in skin, fat and muscles)(Gefen, 2010). Monitoring internal strains is currently a consensual criterion to assess the risk of pressure ulcer and has been widely used in previous studies (Linder-Ganz et al., 2008)(Oomens et al., 2003). Nevertheless, measuring internal strains in vivo being impossible, a biomechanical model integrating the behavior of the foot internal soft tissues and bones is needed to assess internal strains and the resulting risk of ulceration. Furthermore because of inter-individual anatomical variability, personalized biomechanical models must be resorted to in order to accurately estimate internal strains and implement an adequate prevention strategy (Gefen, 2010)(Luboz et al., 2015)(Tenenbaum et al., 2013). 
Several studies have demonstrated the use of biomechanical foot models to estimate internal strains. Most of the feasibility studies are limited to a single foot model generated for a specific patient, and seem difficult to extend in an automatic fashion to a wider group of subjects - not to mention - in clinical routine. For example, (Ledoux et al., 2004) modelled the soft tissues under the foot (skin, fat and muscles) as a Finite Element (FE) mesh with a homogeneous linear elastic material, bones as rigid FE meshes; joints were accounted for as idealized contacts between bones, and around 20 ligaments connecting the mid foot bones were modelled as cables. In another study, (Chen et al., 2010) proposed a more detailed FE foot model including almost all foot ligaments and using a large deformation Mooney Rivlin constitutive law for the soft tissue bulk. Even though this model is fairly complete, it lacks computational efficiency and does not distinguish between different tissue types. These drawbacks were addressed in the model that we recently proposed (Perrier et al., 2015) with foot soft tissues represented as four different Neo Hookean materials for skin, fat, heel pad fat, and muscles respectively. In this model, bones were represented as rigid bodies connected by the most significant ligaments of the foot, modeled as cables. Nevertheless, this last model, just like the two previously cited ones, was generated from a single subject dataset and is consequently only representative of this particular morphology. In this paper, inspired by our previous study on patientspecific modelling of the calcaneus (Luboz et al., 2015), we propose to use this complex foot model as an atlas - or generic model - and to generate new patient-specific models by deforming this atlas to fit the patients' specific morphology. The goal is to design a process making it possible to produce patient-specific biomechanical models in the most automated and user-friendly way possible. The proposed modeling technique could be used to study the influence of variability in morphology on pressure ulcer formation. Its further goal is to provide insight at how morphological specificities should be accounted for in the design of medical devices to optimize strain monitoring-based prevention for each individual. The following study has been carried out in a static analysis framework i.e. does not take into account the duration or repetitive mechanisms leading to pressure ulcer but only tissue compression resulting from a static stance.

\section{Methods}

\subsection{Foot model atlas}

The shape of the atlas model is based on a single subject (male, 33, healthy) and is presented in details in (Perrier et al. 2014a)(Perrier et al., 2015). The contours of the skin, heel fat pad, muscles, and bones were manually segmented from the CT scan of the right foot of this healthy subject. An automatic FE mesh generator (developed by Texisense) was run on the resulting surfaces, and produced a conforming multi-domain FE mesh containing four layers: muscles, fat, heel fat pad, and skin (Figure 1). The meshing algorithm generates as many hexahedrons as possible in the core of the continuum to limit the locking effect observed for tetrahedral elements under quasi-incompressible assumption. Smooth and conforming boundaries between the different internal domains are defined using transition elements such as pyramids, wedges, or tetrahedrons. The meshing procedure led to a FE mesh having 44,220 elements, including 3,610 hexahedrons, 12,062 pyramids, 8,674 wedges, and 19,874 tetrahedrons, for a total of 19,574 nodes.

Finite Element analyses are carried out on the 3D simulation platform ArtiSynth (Lloyd et al., 2012)(www.artisynth.org). Soft tissues (skin, fat, and muscles) are modelled using Neo Hookean materials in order to account for large deformations. Each layer is assigned distinct material properties, 
drawn from literature (Sopher et al., 2011). Young moduli were set to $200 \mathrm{kPa}$ for the skin, $60 \mathrm{kPa}$ for the muscles, $100 \mathrm{kPa}$ for the heel fat pad, and $30 \mathrm{kPa}$ for the rest of the fat. Assuming these tissues are quasi-incompressible, we set the Poisson ratios to 0.485 for the skin, 0.495 for the muscles, 0.499 for the heel fat pad, and 0.49 for other fatty tissue. Bones, featuring a significantly higher stiffness, are modeled as rigid bodies and their shapes are cut out within the soft tissue continuum, i.e. without any finite element inside the volume. The foot 28 bones, the tibia, and fibula are integrated in the model. Each bone can collide with its neighbors and is connected to them by several ligaments, forming the joints. FE nodes nearby bony surfaces are automatically attached to the neighboring bones, which results in a non-sliding soft tissue-bone interface. The main musculoskeletal structures are modeled by active cables elements within the Artisynth framework.

The numerical foot model is divided in two components. The first component is a musculoskeletal model accounting for rigid body motion within the foot. This model implements anatomical constrains such as contacts between adjacent bones, action of the ligaments, or simulated muscle contractions. The second component is the soft tissue continuum modeled by the FE mesh which is iteratively coupled with the musculoskeletal component and translates boundary conditions and internal rigid body motion into elastic deformation in soft tissues.

\subsection{Patient data}

In order to generate patient-specific models, a description of the patient's morphology is needed and more specifically the contours of bones as well as the external skin surface. These shapes are used by the atlas-to-patient registration procedure in order to compute the anatomical transfer of musculoskeletal data. This three-dimensional information can be provided by various sources such as CT, MR scans, or EOS images (provided a 3D reconstruction of the morphology is performed by the latter bi-planar imaging device). Extracting tissue contours from these modalities involves specific automatic or semi-automatic procedures and is a challenge in itself that lies beyond the scope of this article. In our study, this task has been carried out manually. In the remainder of the article, we describe our workflow assuming that the medical images are already labelled (i.e. segmented), forming so-called "binary images". Each label in a binary image represents a distinct bone or soft tissue, which will be implemented as a modeling domain in subsequent mesh generation steps.

Three patients were included in our study: a 70 year old male (BR), a 67 year old male (FP) and a 55 year old male (FC). Images were acquired while the patients were in dorsal decubitus, legs and feet supported by the table. The exams were performed in the context of a vascular exam using CT angiography. Clinical exam revealed that the feet in all three patients were healthy. The size of the CT volumes is as follows: $220 \times 404 \times 519$ for BR, 196x276x575 for FC, and 183x297x580 for FP, and the resolution is $1 \times 1 \times 1 \mathrm{~mm}^{3}$ in all three datasets. The right foot is modeled for both FC and FP, and the left foot for BR.

\subsection{Patient-specific model generation}

Shapes of patient's bones and skin were recovered from binary images. Bony surfaces were directly used as rigid bodies in the numerical model. Each patient's personalized musculoskeletal model was generated by registering the patient's bones and skin with their counterparts in the atlas. The Mesh-Match-and-Repair (MMRep) registration algorithm is used to perform this task automatically in about a minute (Bucki et al., 2010). Our implementation of the atlas to patient 
registration procedure is divided into three steps increasingly introducing distortion in the data: (1) a rigid registration that roughly positions the patient data set with respect to the atlas model, (2) an affine deformation that compensates for global scale discrepancies, followed by 3) an elastic registration that accurately fits the bony contours and the skin surface. Once all three deformation functions are combined, the resulting deformation is applied to the atlas dataset to transfer the atlas information (muscles, ligament insertions, fat pad, plantar fascia) into the patient's referential. The procedure - producing the musculoskeletal component of the patient model - is automatic and takes about two minutes.

The assembly of the soft tissue continuum mainly consists of the generation of the FE mesh corresponding to the fat, muscle, fat pad and skin domains. The outlines of these domains however are not present in the binary images in our dataset. Indeed, for the sake of integration of our procedure within a realistic clinical workflow, we assumed that only a basic segmentation could be performed to extract the prominent morphological features that are the bones and skin. In order to overcome this practical hurdle, we again resort to the atlas approach to infer the missing information in the patient data. The volumetric deformation function computed using the MMRep algorithm for the tendon and ligament insertions is applied to the soft tissue domains defined in the atlas that we wish to replicate in the patient's model. The outlines (materialized by triangular surface meshes) of both the muscles and fat pad are deformed and their position is adjusted to fit the patient's bones and skin. Then Texisense mesher algorithm is used to automatically produce a conforming FE mesh of the domains. This step takes about three minutes. Before the mesh generation, a preprocessing step for the selection of the region of interest containing the foot (simple cropping of the image around the skin and bones) is performed manually and requires about 10 minutes of user intervention. The definitions of the muscle and fat pad subdomains in the patient model rely on assumptions formulated in the atlas as well as approximations involved by the registration procedure. However, in the current state of the art, we believe that the atlas paradigm provides the right balance between the efficiency of existing image processing techniques and a level of accuracy required for the targeted biomechanical simulation in the context of pressure ulcer prevention. In the future, should a new segmentation algorithm (or a new imaging modality) appear that would enable an accurate and cost effective segmentation of one or all of these domains, our approach could easily take advantage of it by replacing our registered domain by the actual one yielded by the novel technique.

Once the FE mesh has been produced, a quality control and optimization step is performed to improve the elements that might put FE analysis in jeopardy. These elements are identified using popular FE quality metrics such as Aspect Ratio and Jacobian Ratio and derivations of such. This step is semi-automatic as an informed user needs to supervise this mesh untangling. However, once the parameters set, mesh relaxation is automatic and takes approximately five minutes.

Lastly, since the atlas is a right foot, plain mid-sagittal plane symmetry flips it to accommodate a left foot in the patient.

The whole above described model specialization process takes less than 20 minutes.

\subsection{Estimation of foot ulcer risk through simulation}

To study the influence of foot morphology on the location and magnitude of internal strains, and therefore on the risk of pressure ulcer, a common pressure pattern simulating a static unipodal stance was applied below all three virtual foot soles. The chosen plantar pressure pattern was measured using a commercially available pressure sensor (Zebris platform, http://www.zebris.de/) 
under the right foot of the subject used to build the atlas (Figure 2). Most of the plantar pressures mainly appear below the heel and the metatarsal heads, with a peak of $14.5 \mathrm{~N} . \mathrm{cm}^{-2}$ below the calcaneus. The pressure pattern has been mirrored prior to applying the boundary conditions on the left foot of patient BR. The pressure map was aligned under the foot by fitting the highest pressure peak below the lowest point of the calcaneus for each patient, which implicitly forms the assumption that this bony prominence is the source of peak pressures under the heel. The axis of the foot was given by the vector pointing from this lowest calcaneus point to the lowest point under the second metatarsal head.

During the simulations, the tibia was fixed while the rest of the foot and the fibula were left free to move. The first phase of the simulations allowed the foot to relax under the influence of the multiple tendons, ligaments and muscles of the model which tend to recover their equilibrium length and thus generate pre-stresses in the FE continuum. These musculoskeletal structures were not initially at their resting length because their morphology is derived from the medical image dataset, and consequently from the pre constrained position imposed upon the patient during the medical exam. Once steady state reached, pressure patterns were projected below the foot and the FE nodes at the surface of the foot model were assigned the pressure corresponding to their position in the pressure pattern. These normal pressure values were converted into nodal forces. Plantar pressure was applied gradually. Once $100 \%$ of the pressure was applied, the loading was maintained until equilibrium was reached. The reported strain values are those measured in the final equilibrium configuration of the models.

\subsection{Cluster analysis and regionalization}

In order to assess the risk of pressure ulcer, besides monitoring the maximal internal strains, we introduce a novel paradigm in our Finite Element simulations by considering the volume of the largest group of adjacent elements with nodes exhibiting a VM strain over one of the considered 20 or $50 \%$ thresholds, as suggested by (Loerakker et al., 2011)for the muscle tissue. We call "clusters" the isolated groups of such adjacent and over-strained elements. Clusters are determined by aggregating all the neighboring elements with strains higher than a given threshold. The external boundary of a cluster is defined as the set of all cluster element faces not shared by another element in the cluster. The shape, volume and hence boundary of clusters depend on each individual's morphology and tissue behavior. A cluster can be heterogeneous in tissue nature which means that fat, muscle, and/or skin elements can share a common cluster. In the absence of results for fatty tissues at the time of this study, we applied the same strain thresholds for the whole soft tissue bulk. Should any future experiments lead to strain threshold values in fat or skin, these parameters could easily be integrated in the cluster definition by merely adjusting each element's inclusion test to the threshold of the tissue it stands for in the model.

One possible interpretation that can be derived from clusters is referred to, herein, as "cluster volume" and is merely the volume (in $\mathrm{mm}^{3}$ ) of the considered cluster. However, other "indicators" i.e. scalar interpretations of these clustered subsets of elements within the continuum mesh - can be contemplated but were not investigated in this study. Let's just mention a few : the extent of a cluster in a specific direction - e.g. in anisotropic tissues, the amount of blood vessels that it encompasses and is likely to influence, the proportion of different kinds of soft tissues within, or its geometrical correlation with the patient's previous lesion history. 
Clusters allow quantitative comparison of tissue suffering levels among individuals while overcoming the lack of reliability of peak VM strains which can locally stem from numerical uncertainty in the FE analysis. The mathematical rationale here is that important jumps in the gradient of the solution (here the displacement field) are a known indicator of local numerical uncertainty in Finite Element analysis (Verfürth, 1999) and it is unwise to draw conclusions from these local epiphenomena.

Cluster analysis however introduces a new unknown which is the "minimal lethal cluster volume" i.e. the value of the volume of tissue above which a pressure ulcer may develop following one of the above-defined thresholds i.e. short term or long term lesion. In the absence of physiological definition of such volume, we are at the moment restricted to relative conclusions which can be formulated as: "this patient is more at risk than that patient, or this insole is better suited for this patient than that insole." Indeed, cluster volume might either indicate the lack of relevance of a strain value if it is associated with a negligible volume (yet to be defined) or, on the contrary, show that the strain value is observed at a macroscopic scale and most likely affects a significant volume of tissue, hence leading to a possible lesion. To rephrase our proposition, the assertion "The maximal volume of

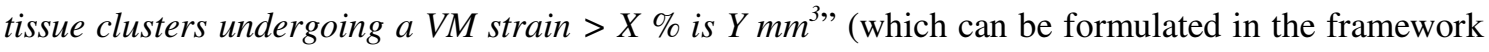
of cluster analysis and interpreted in a comparative study) is more intuitive and numerically more robust than the assertion "The maximal VM strain in the model is Z\%."

Cluster localization within the foot volume also provides information on the areas where lesions are prone to appear. To make the interpretation of the results more intuitive and clinically relevant the foot was partitioned into eight key anatomical regions defined as follow (Figure 3): (1) the Achilles tendon, (2) the top of the foot, (3) the heel, (4) the medial foot, (5) the first metatarsus, (6) the four other metatarsi, (7) the hallux, (8) and the four other toes. This partitioning makes it possible to correlate cluster volumes with their respective locations within the foot anatomy which in turn relates to corresponding foot functions that might be affected by a potential lesion. A risk level per region can thus be assessed and used to refine a prevention strategy or the design of an orthosis. The foot regions are defined within the atlas and are automatically adjusted to each patient's foot using the MMRep inference procedure described above.

\section{Results}

Finite Element meshes for the atlas model and the three studied patients are presented in Figure 4. Morphological differences between the three patients are obvious: various external foot shapes, and various individual bone shapes and orientations. The main difference in terms of external shape is around the phalanges of subject FC compared to subjects FP and BR, and even though moderately, with the atlas. The ankles of the atlas and subject FC are also quite different in shape compared to subject FP and BR, which are more prominent. In terms of internal morphology, BR has very narrow metatarsal bones compared to the other subjects. The talus of the atlas and subject $\mathrm{FC}$ are wider than the ones of subjects FP and BR. The navicular of the atlas and subject FC are located more medially than the ones of subjects FP and BR. The calcaneus of subject FC and BR are curved more medially than the ones of subject FP and the atlas.

The maximal Von Mises strains and cluster volumes for the atlas and patients are summarized in Table 1 (for a threshold of VM strains over 20\%, representing long term lesion) and Table 2 (for a threshold of VM strains over 50\%, representing short term lesion). For each patient (atlas, FC, FP and 
BR) the table is split vertically in two columns: maximal cluster volume for the considered VM strain (20\% in table 1,50\% in table 2) and maximum VM strains inside that maximum cluster volume. Both tables are divided horizontally providing detailed strain information for each of the eight anatomical regions. The mean and standard deviation are also provided for the eight regions.

For the atlas, it can be seen that the highest cluster volume is located in the heel region $(50.8$ $\mathrm{cm}^{3}$ with VM strains above $20 \%$ ). As for the VM strains, the highest values are located in region 6 comprising the second, third, fourth and fifth metatarsi (161\%), the top of the foot region (145\%), and the medial foot region (107\%). These values are highlighted in Figure 5, which illustrates, in a color code, the VM strains and the cluster volumes region by region. For each foot, the maximum value is coded as red and the minimum is blue. In Figure 5, the Achilles and top of the foot regions are not shown as the regions mostly at risk under plantar compression are below the foot. Figure 6 shows the differences in locations of the maximal VM strain and the largest cluster volume, compared to the calcaneus bone.

For patient FC, the maximum VM strain $(348 \%)$ is located in region 5 with the first metatarsal bone, while the maximum cluster volume $\left(74.4 \mathrm{~cm}^{3}\right.$ for VM strains above $\left.20 \%\right)$ is in the heel region. For patient FP, these regions become the top of the foot region with values of $399 \%$ (which is a numerical singularity due to a node compressed between two bones: the calcaneus and the cuboid) for the maximum VM strain and the medial foot region with a maximum cluster volume of $62.2 \mathrm{~cm}^{3}$ (for VM strains above $20 \%$ ). Note that for this patient, a second region is highly at risk: the heel region, as it reaches a cluster volume of $57.5 \mathrm{~cm}^{3}$ (for VM strains above $20 \%$ ). For patient BR, the maximum VM strains point to the medial foot region with $205 \%$ for a high risk of pressure ulcer while the cluster volumes again highlight the heel region $\left(63.0 \mathrm{~cm}^{3}\right.$ for $\mathrm{VM}$ strains above $\left.20 \%\right)$ as most at risk. Note that the cluster volumes for the medial region is only $14.1 \mathrm{~cm}^{3}$, therefore corroborating that this region is less at risk than the heel region.

A similar analysis can be made for all datasets using Table 2 and Figure 5 based on the VM strain threshold of $50 \%$.

\section{Discussion}

For the same pressure pattern applied below the foot of each patient, the variability of the results among patients presented in Tables 1, 2 and in Figure 5 clearly point out the influence of the patient's morphology. Whereas the simulations report a huge range of variations for maximum VM strains (between 161 to $399 \%$ from one patient to the other, with almost all foot regions affected, including the upper part of the foot which seems not relevant), the values reported for the maximum cluster volumes seem more coherent. Indeed, except a specific case (patient FP who has a medial foot maximal cluster volume barely higher than for the heel), the maximal cluster volume is located in the heel region for all patients, with values ranging between 50.8 and $74.4 \mathrm{~cm}^{3}$ (for VM strains above 20 $\%$ ) and between 0 and $21.6 \mathrm{~cm}^{3}$ (for VM strains above $50 \%$ ).

These results seem therefore to show that only monitoring the maximum VM strain is probably not the best option to evaluate the risk of pressure ulcer and its location. This observation seems similar to the one stemmed from another study on buttocks' ulcer analysis (Luboz et al., 2014). The non-realistic strain value of $399 \%$ reported for patient FP is a good example of numerical "outliers" that can be generated by any FE model submitted to high pressures. This high value strain singularity is located at only one node that appears to be squeezed between the cuboid and calcaneus bones; the element associated to that node is therefore deformed a lot because of the mesh 
configuration and not because of the pressure applied below the foot. Furthermore, the high VM strains reported in the top of the foot (regions 1 and 2) for the atlas, patient FP and patient BR also show the limitations of using this criterion as high strain values should not be located in the top of the foot during a plantar load. Such singularities can be ignored by analyzing the maximum cluster volumes. Indeed, this analysis checks if the maximum strain is located at a single node (or few nodes), by computing the volume associated to contiguous elements exhibiting a strain over a given threshold. Outliers such as the one observed in our simulations will thus automatically be ignored by the cluster analysis since they only affect a much reduced volume of tissues. This is demonstrated especially for the atlas and patient FP where the heel region is not described as at risk by the maximal VM strain analysis while it is clearly at risk for the cluster volume one.

Given the variety of bone shapes observed in our small sample, see Figure 6 for an example on the calcaneus shape, we think that an accurate representation of the internal structures is necessary to capture accurately the behavior of soft tissues under compression for an articulated foot. A non-linear registration step is therefore compulsory in order to transfer the anatomical knowledge from the atlas to the patient. Using only steps 1 and 2 (rigid and affine) in the MMRep procedure described in paragraph 2.3 would result in an approximate deformation that is likely to miss morphological specificities that can possibly result in an injury, see Figure 6 for the location of the strains above the threshold of $20 \%$ below the calcaneus of each patient. This is particularly true in the case of strongly pathological feet exhibiting large differences with the atlas mesh.

Another important conclusion is that, consistently with other pressure ulcer prevention studies (Gefen, 2003)(Gefen, 2010)(Luboz et al., 2014)(Luboz et al., 2015), it appears impossible to build a pressure ulcer risk assessment scale by relying solely on interface pressures. The compression of soft tissues, with patient dependent thicknesses and parameters, under personalized bony prominences is key to an efficient personalized prevention strategy. This is for example the case for the medial foot region of patient FP which is subject to the same pressure as the other patients but turns to be at risk for patient FP and not for the others.

A large variability of results has been observed on a small sample $(\mathrm{N}=3)$ of patients and these trends would most likely be confirmed on a larger sample.

One limitation of our models is probably the fact that the same generic tissue constitutive law was proposed for each patient. The Neo Hookean law was chosen to simulate the quasiincompressibility of the soft tissues and to account for large deformations. The Young moduli of each tissue (muscle, skin, fat and fat pad) are based on the literature, as introduced in section 2.1. We assumed the same constitutive parameters to avoid hindering the influence of the anatomy in this study. However, the variations of the soft tissues properties would need to be specified for each patient, for example by using indentation or elastography techniques.

Another limitation identified during this study is the difficulty to compute a proper non-linear registration between the atlas and patient toes. Indeed, the differences between the toes' posture combined with the complexity of the shape drive the elastic registration algorithm towards a local minimum. Registration accuracy in this region is thus compromised as can be seen in Figure 5 where the toes in patients exhibit an unnatural distortion (see e.g. the fifth toe of patient FP and the first toe of patient BR). However, this phenomenon has only a regional effect and does not influence the results observed in other regions thanks to the realistic stress smoothing introduced by the foot tendons and ligaments included in our model and the different layers of material properties modelling the skin, fat 
and muscles. The discussion carried out on the heel and medial foot thus retains its relevance. An accurate registration in the toe region will require the individualization of each toe in order to rewrite the objective function used in MMRep to compute the volumetric deformation.

Finally, it must be noted that our comments are based on a static analysis (unipodal stance) while pressure ulcers can appear while walking. Gait can be decomposed into a number of foot positions on the ground and their corresponding pressure maps. Applying these pressure maps under the foot model would make it possible to simulate the deformation of the foot at each gait phase. It would therefore be possible to perform a dynamic analysis and estimate the risk of pressure ulcer at these stages exactly as it is done in this paper for unipodal standing alone.

In order to implement an efficient pressure ulcer prevention strategy, daily monitoring of plantar pressures underneath the foot (but also above the toes and wherever lesions may develop) is necessary, and as the present study points out, it also needs to be coupled with a predictive and personalized biomechanical model of the foot. Although plantar pressure monitoring is feasible in a laboratory or clinical setting using a heavy and expensive pressure sensor (such as the Zebris platform used in this article), it is impossible to implement for a large number of patients on a daily basis. To achieve this goal, a lighter and less expensive pressure sensor (such as the ones proposed by Novel, Tekscan, Vista Medical or Texisense) able to monitor the patient's foot pressures in his/her daily tasks must be used. For example, using a technology similar to that recently employed for the conception of the TexiCare device dedicated to the prevention of seated buttock pressure ulcers for people with spinal cord injury (Chenu et al., 2013), a "Smart Sock" (Bucki et al., 2011)(Perrier et al. 2014b), has been developed. It is made of a $100 \%$ textile pressure sensing fabric wirelessly connected to a controller which can record and monitor the pressures all around the foot (not only under the sole). It can be used continuously during everyday activities. Once coupled to personalized biomechanical models such as the ones presented here, this device could be used to estimate the internal strains and to raise an alert, should the risk factor exceed a predefined personalized threshold. The main limitation at the moment lies in the implementation on a mobile platform of the complex biomechanical models such as the ones described here, where large non-linearities (mechanical, geometrical, contacts) need to be taken into account. At the moment the simulations require approximately three hours to converge to a steady state for a single pressure pattern applied to the foot sole. Our team is working towards drastically reducing the computational complexity of the models - while retaining most of their accuracy - in order to bring this technology into the clinical practice and benefit to the largest number of users: personalized prevention algorithms seamlessly embedded in wearable devices.

\section{Conclusion}

A workflow for generating a patient-specific biomechanical model of the foot has been presented and evaluated in this article in the context of pressure ulcer prevention. The technique implements an atlas based approach where anatomical knowledge is automatically transferred to the patient's modeling space using a non-linear registration algorithm. A new paradigm for the assessment of the level of tissue suffering in the context of pressure ulcer prevention has been proposed. It is based on the most recent consensus which relies on the measurement of Von Mises equivalent strains. The paradigm suggests looking at the volumes of "clusters" of elements undergoing a deformation greater than a predefined threshold (20\% and $50 \%$ in the literature). This approach eludes the erratic 
results yielded by the monitoring of maximal VM strain values and opens the way to comparative assessment of a risk score that can be used to drive medical device design or clinical studies on a given population. The new paradigm however raises a new question which is "what is the minimal volume of tissue undergoing damage that is likely to lead to a lesion?" The answer to this question is beyond the scope of this study and will most likely not be a single figure but rather a threshold to be investigated based on the nature of the tissues (muscle or fat), the clinical condition and history of the patient, and other extrinsic factors that still have to be identified.

The approach was assessed on three patients and demonstrates the feasibility of patientspecific model generation. The evaluation was carried out by simulating the deformation of the personalized biomechanical models under the influence of a static common pressure pattern applied below the foot and by measuring the resulting internal strains. The results were further regionalized by dividing the foot into eight functionally meaningful regions. The results indicate that, for the chosen pressure pattern, the main risk of pressure ulceration is located below the heel for all four datasets (three patients and the atlas). The analysis shows that cluster analysis is an interesting alternative to the peak VM strain alone (as this value is strongly affected by numerical uncertainties inherent to numerical methods) and could be used to predict the risk of pressure ulcer and its localization within the foot regionalized representation. The study also confirms the influence of the patient's morphology on the range of the VM strains and associated cluster volumes: for the same pressure pattern, various values are obtained for both criteria, on all four datasets.

Before implementing this pressure ulcer prevention technique in a clinical workflow, some aspects of the approach still require improvement: the personalization of the patient's material properties for the various soft tissues layers, the precision of the registration on the toes, and the measurement of the pressure below the foot using a flexible textile sensor in real time to allow the patient to use this prevention tool on a daily basis.

\section{Acknowledgments}

Competing interests: Some authors are involved with the TexiSense Company (http://www.texisense.com/home_en).

Funding: This work was partly funded by the 2010 ANR TecSan IDS project, by the CAMI Labex (ANR-11-LABX-0004) and by the Institut Universitaire de France.

Ethical approval: the study was approved by the French institutional review board of Grenoble (IRB 5891 (CECIC) for Rhône-Alpes-Auvergne).

\section{References}

1. Atlas E., Yizhar Z., Khamis S., Slomka N., Hayek S. \& Gefen A. Utilization of the foot load monitor for evaluating deep plantar tissue stresses in patients with diabetes: Proof-of-concept studies. Gait \& Posture, 2009; 29:377-382.

2. Boulton A.J.M., Vileikyte L., Ragnarson-Tennvall G. \& Apelqvist J. The global burden of diabetic foot disease. The Lancet, 2005, 366:1719-1724.

3. Bucki M., Lobos C., \& Payan Y. A Fast and Robust Patient Specific Finite Element Mesh Registration Technique: Application to 60 Clinical Cases. Medical Image Analysis, 2010, 14:303-317.

4. Bucki M., Vuillerme N., Cannard F., Diot B., Becquet G. \& Payan Y. The TexiSense « Smart Sock » - Textile Pressure Sensor and 3D Real-time Finite Element Model of the Diabetic Foot for a Daily Prevention of Pressure Ulcers. Proceedings of the 14th Annual European Pressure Ulcer Meeting, EPUAP, 2011. 
5. Chen W.M., Lee T., Vee-Sin Lee P. \& Lee S.J. Effects of internal stress concentrations in plantar soft-tissue - preliminary three-dimensional finite element analysis, Medical Engineering \& Physics., 2010, 32:324-331.

6. Chenu O., Vuillerme N., Bucki M., Diot B., Cannard F. \& Payan Y. TexiCare: An innovative embedded device for pressure ulcer prevention. Preliminary results with a paraplegic volunteer, Journal of Tissue Viability, 2013, 22, pp. 83-90.

7. Gefen A. Plantar soft tissue loading under the medial metatarsals in the standing diabetic foot, Medical Engineering \& Physics, 2003; 25:491-499.

8. Gefen A. The biomechanics of heel ulcers. Journal of Tissue Viability, 2010, 19, 124-131.

9. Gordois A, Scuffham P, Shearer A, \& Oglesby A. The healthcare costs of diabetic peripheral neuropathy in the UK. Diabetic Foot, 2003; 6: 62-73.

10. Kosiak M. Etiology and pathology of ischemic ulcers. Archive of Physical Medicine and Rehabilitation, 1959; 40: 62-69.

11. Ledoux W.R., Meany D.F. \& Hillstrom H.J. A quasi-linear, viscoelastic, structural model of the plantar soft tissue with frequency sensitive damping properties. Journal of Biomechanical Engineering, 2004, 126:1-7.

12. Linder-Ganz E., Shabshin N., Itzchak Y., Yizhar Z., Siev-Ner I. \& Gefen A. Strains and stresses in sub-dermal tissues of the buttocks are greater in paraplegics than in healthy during sitting. Journal of Biomechanics, 2008, 41:567-580.

13. Lloyd J.E., Stavness I. \& Fels S. ArtiSynth: a fast interactive biomechanical modeling toolkit combining multibody and finite element simulation. Soft Tissue Biomechanical Modeling for Computer Assisted Surgery, Studies in Mechanobiology, Tissue Engineering and Biomaterials, Springer, 2012; 11:355-394.

14. Loerakker S., Manders E., Strijkers G.J., Nicolay K., Baaijens F.P.T., Bader D.L., et al. The effects of deformation, ischaemia and reperfusion on the development of muscle damage during prolonged loading. Journal of Applied Physics, 2011. 111(4): 1168-1177.

15. Luboz V., Petrizelli M., Bucki M., Diot B., Vuillerme N. \& Payan Y. Biomechanical Modeling to Prevent Ischial Pressure Ulcers. Journal of Biomechanics, 2014, 47: 2231-2236.

16. Luboz V., Perrier A., Bucki M., Diot B., Cannard F., Vuillerme N., \& Payan Y. Influence of the Calcaneus Shape on the Risk of Posterior Heel Ulcer Using 3D Patient-Specific Biomechanical Modeling. Annals of Biomedical Engineering, 2015, 43(2): 325-335.

17. Mueller MJ. Etiology, evaluation, and treatment of the neuropathic foot. Critical Reviews of Physical and Rehabilitation Medicine, 1992; 3: 289-309.

18. Oomens C.W.J., O.F.J.T. Bressers, E.M.H. Bosboom, C.V.C. Bouten, \& Bader D.L. Can Loaded Interface Characteristics Influence Strain Distributions in Muscle Adjacent to Bony Prominences? Computer Methods in Biomechanics and Biomedical Engineering, 2003. 6(3):171-180.

19. Perrier A., Luboz V., Bucki M., Cannard F., Vuillerme N. \& Payan Y. Evaluation of a musculoskeletal finite element model of the foot. Proceedings of the Computer Methods in Biomechanics and Biomedical Engineering conference (CMBBE), 2014.

20. Perrier A., Vuillerme N., Luboz V., Bucki M., Cannard F., Diot B., Colin D., Rin D., Bourg J.P., \& Payan Y. Smart Diabetic Socks: Embedded device for diabetic foot prevention. Innovation and Research in BioMedical engineering (IRBM), 2014. 35:72-76.

21. Perrier A., Luboz V., Bucki M., Vuillerme N., \& Payan Y. Conception and evaluation of a 3D musculoskeletal finite element foot model. Computer Methods in Biomechanics and Biomedical Engineering, 2015, 18(1):2024-2025.

22. Shaw J.E., Boulton A.J.M. The pathogenesis of diabetic foot problems. An overview. Diabetes 1997; 46 (2):S58-S61.

23. Shearer A., Scuffham P., Gordois A., \& Oglesby A. Predicted costs and outcomes from reduced vibration detection in people with diabetes in the US. Diabetes Care, 2003; 26: 230510 . 
24. Sopher R., Nixon J., McGinnis E. \& Gefen A. The influence of foot posture, support stiffness, heel pad loading and tissue mechanical properties on biomechanical factors associated with a risk of heel ulceration, Journal of Mechanical Behavior in Biomedical Materials, 2011, 4(4): 572-82.

25. Telfer S., Erdemir A., Woodburn J., \& Cavanagh P. R. What Has Finite Element Analysis Taught Us about Diabetic Foot Disease and Its Management? A Systematic Review, PLoS ONE, 2014, 9(10).

26. Tenenbaum S., N. Shabshin, A. Levy, A. Herman, and A. Gefen. Effects of foot posture and heel padding devices on soft tissue deformations under the heel in supine position in males: MRI studies. Journal of Rehabilitation Research and Development, 2013, 50(8):1149-56.

27. Van Schie C.H.M. \& Boulton A.J.M. Biomechanics of the Diabetic Foot, The Road to Foot Ulceration. In The Diabetic Foot, Second Edition. Veves V., Giurini JM and LoGerfo FW Editors, Humana Press Inc., Totowa, NJ, 2006.

28. Verfürth R. A review of a posteriori error estimation techniques for elasticity problems. Computer Methods Applied to Mechanical Engineering. 1999, 176: 419-440. 
Figure captions:

555 Figure 1 - Cross section of the FE mesh representing the foot soft tissues: plantar skin layer, 556 muscle layer, and the fat in-between. The white sections represent the locations of the rigid bodies 557 modeling the bones.

558 Figure 2 - Distribution of pressures applied under all foot models used in the study. The highest 559 recorded pressures $\left(14.5 \mathrm{~N} . \mathrm{cm}^{-2}\right)$ appear in red (below the heel). Lower values are shown in blue.

560 Figure 3 - The eight anatomical regions defined to partition our foot model: (1) the Achilles 561 tendon, (2) the top of the foot, (3) the heel, (4) the medial foot, (5) the first metatarsus, (6) the four 562 other metatarsi, (7) the hallux, (8) and the four other toes.

563 Figure 4 - The three personalized biomechanical models are derived from the atlas model using the 564 MMRep algorithm which computes a non-linear correspondence function between atlas and patient 565 anatomical landmarks.

566 Figure 5 - Maximum strain and maximum cluster volume repartition for each anatomical region 567 and for each patient, according to the two strain thresholds of 20 and $50 \%$. For the strains above a 568 threshold of $20 \%$ and above a threshold of $50 \%$, red colors mean strains above $200 \%$. For cluster 569 volume above a threshold of $20 \%$, red means volume above $74 \mathrm{~cm}^{3}$. And for a cluster volume above a 570 threshold of $50 \%$, red means volume above $6 \mathrm{~cm}^{3}$.

571 Figure 6 - Location of the strains above the threshold of $20 \%$ below the calcaneus of each patient 572 (a blue color represents strains close to $20 \%$ while red is the maximum, around $110 \%$ ). The 573 morphological variation from one patient to another can also be observed.

Table 1 - Maximal Von Mises (VM) strains (in \%) in the cluster of maximum volume and cluster 576 volumes (in $\mathrm{cm}^{3}$ ) above $20 \%$ for the atlas and the three considered patients (FC, FP and BR). The 577 eight rows in the table correspond to the eight anatomical regions. The mean and standard deviation 
578 (in percentage points, ppt) for these eight regions are shown for both maximal VM and maximal 579 cluster volumes.

580 Table 2 - Maximal Von Mises (VM) strains (in \%) in the cluster of maximum volume and cluster 581 volumes (in $\mathrm{cm}^{3}$ ) above $50 \%$ for the atlas and the three considered patients (FC, FP and BR). The 582 eight rows in the table correspond to the eight anatomical regions. The mean and standard deviation 583 (in percentage points, ppt) for these eight regions are shown for both maximal VM and maximal 584 cluster volumes. 


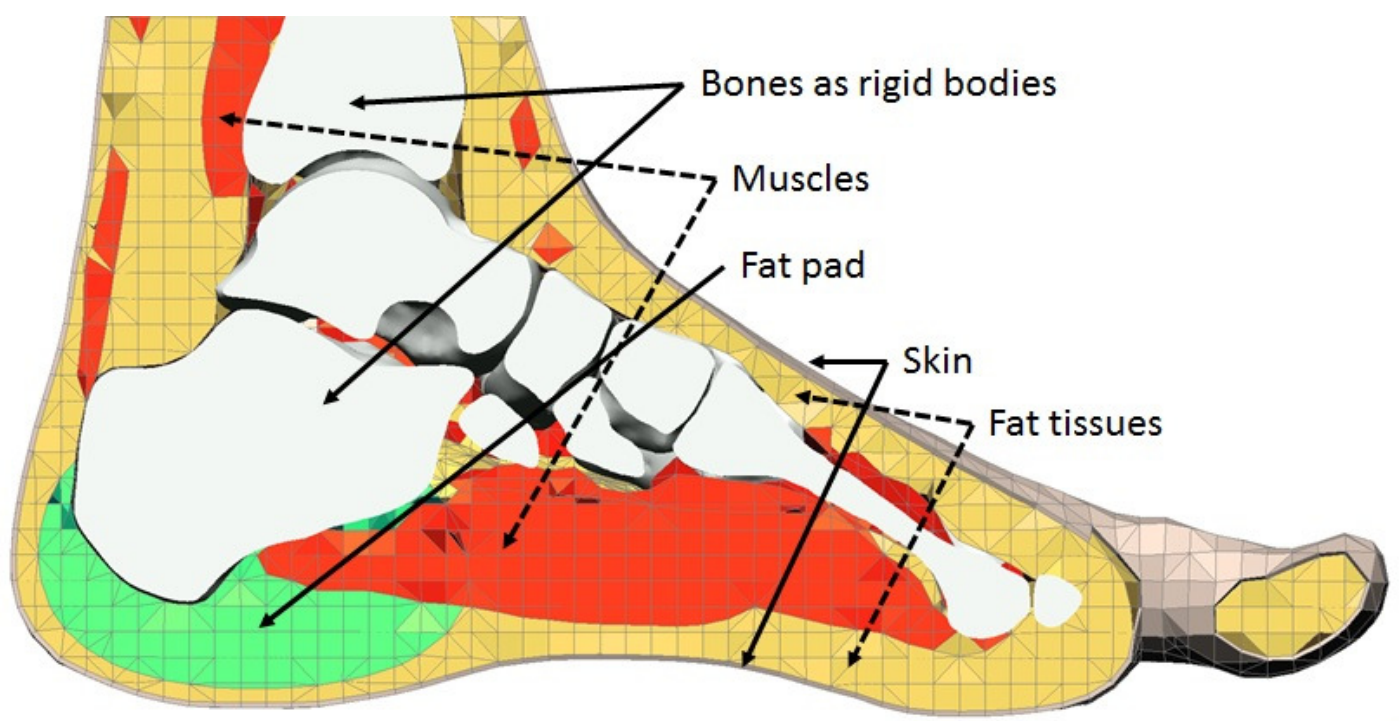


Figure 2

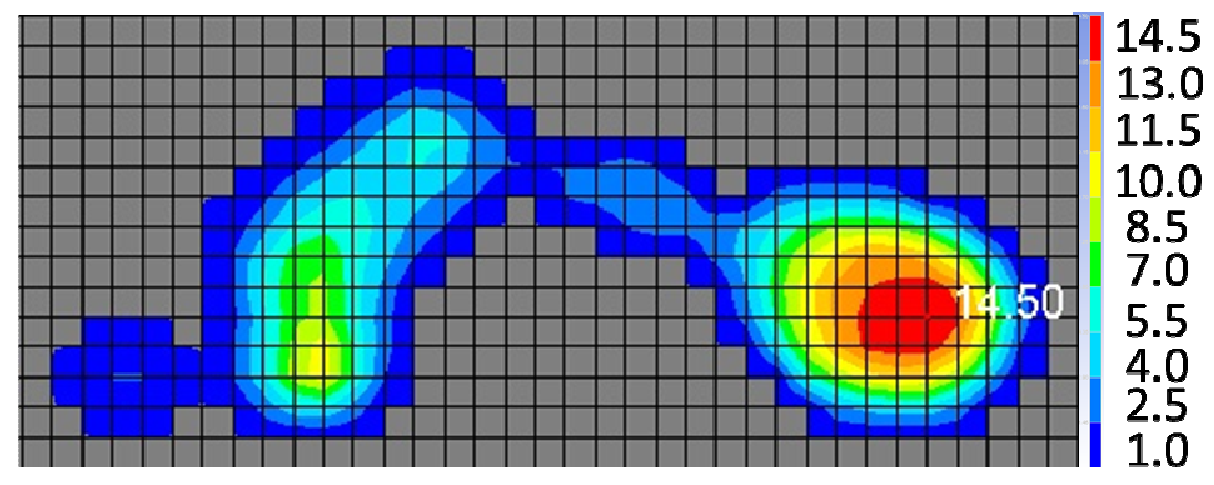

590

591

592 
$593 \quad$ Figure 3
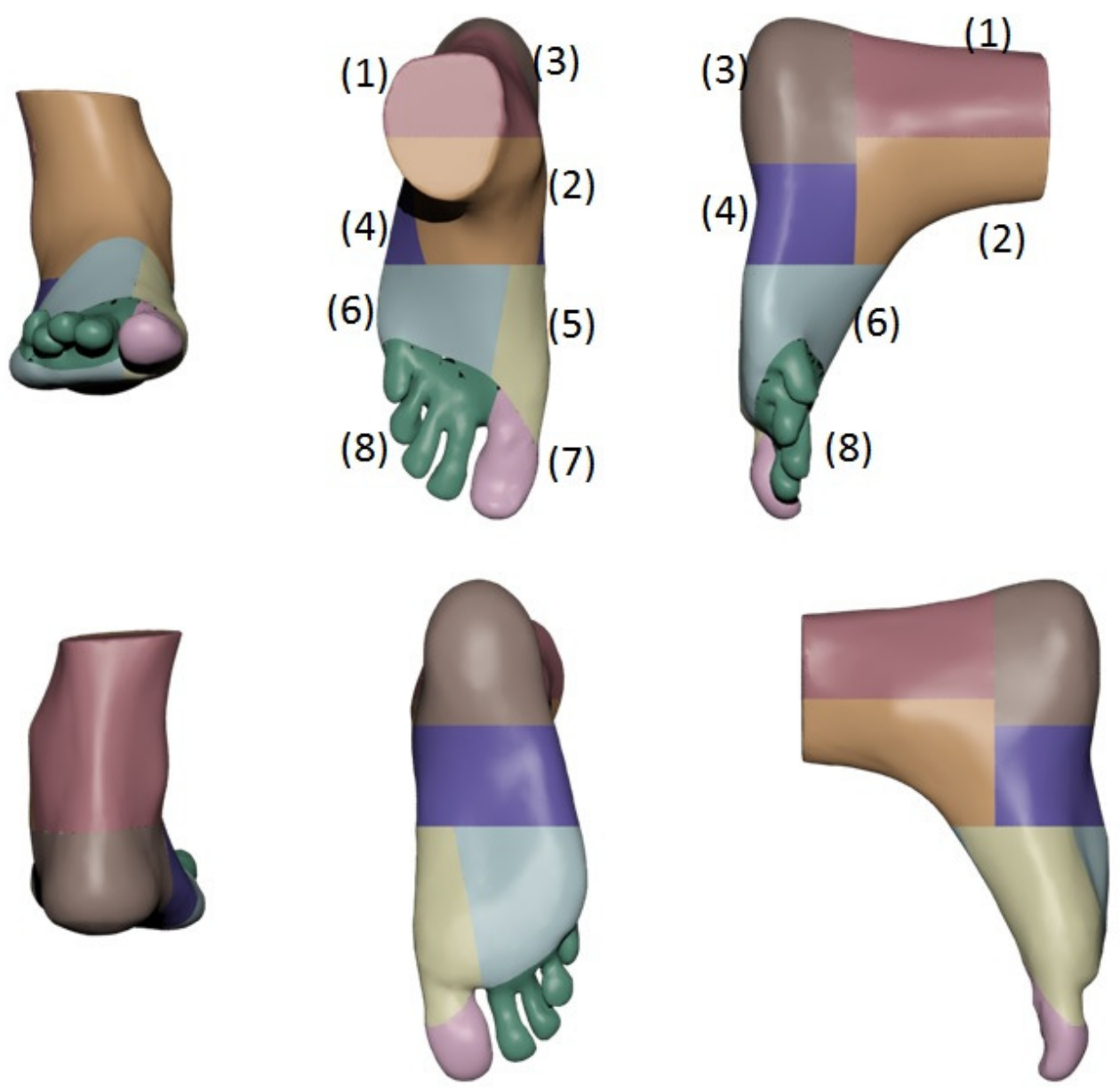

594

595

596

597 
Figure 4

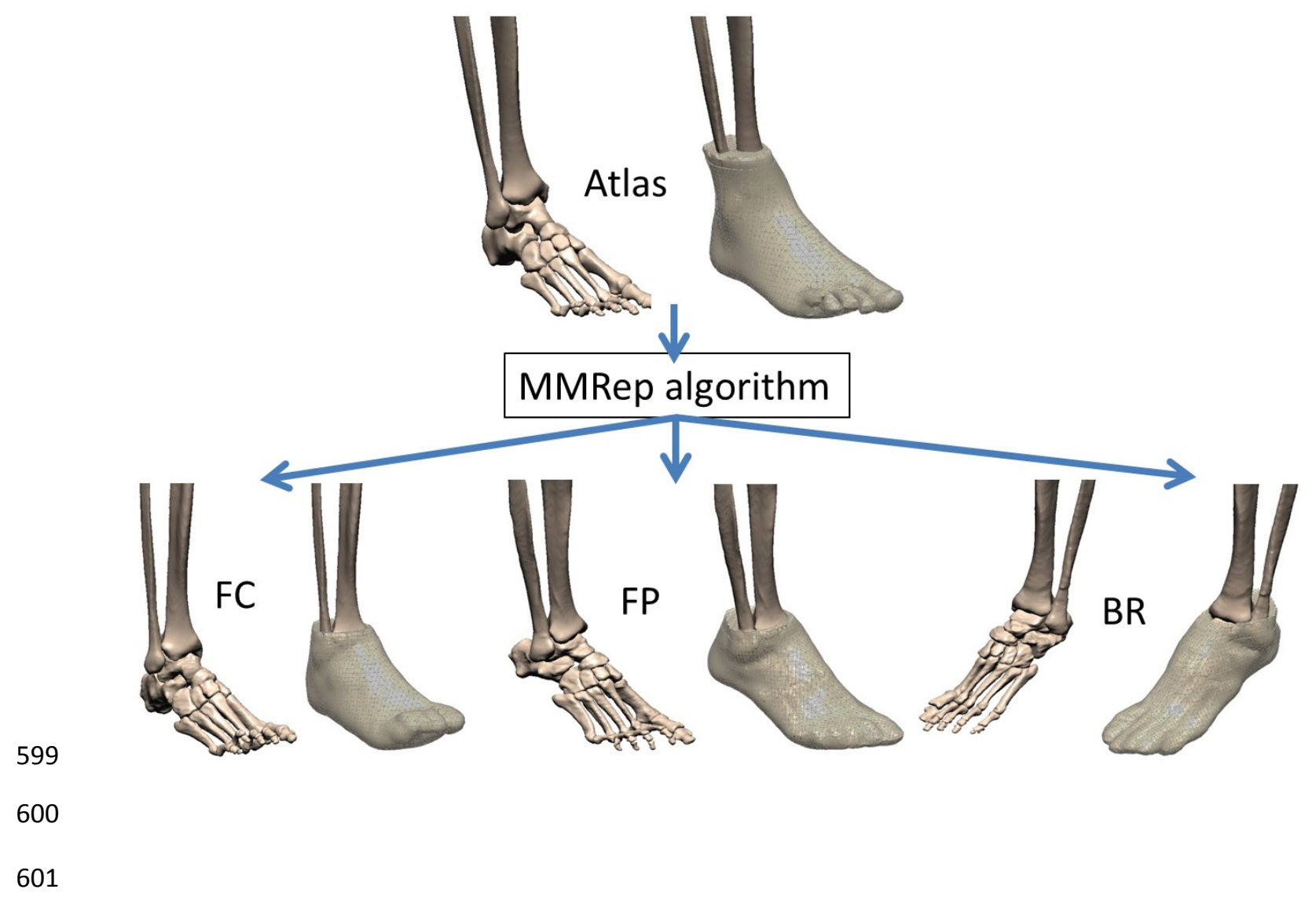




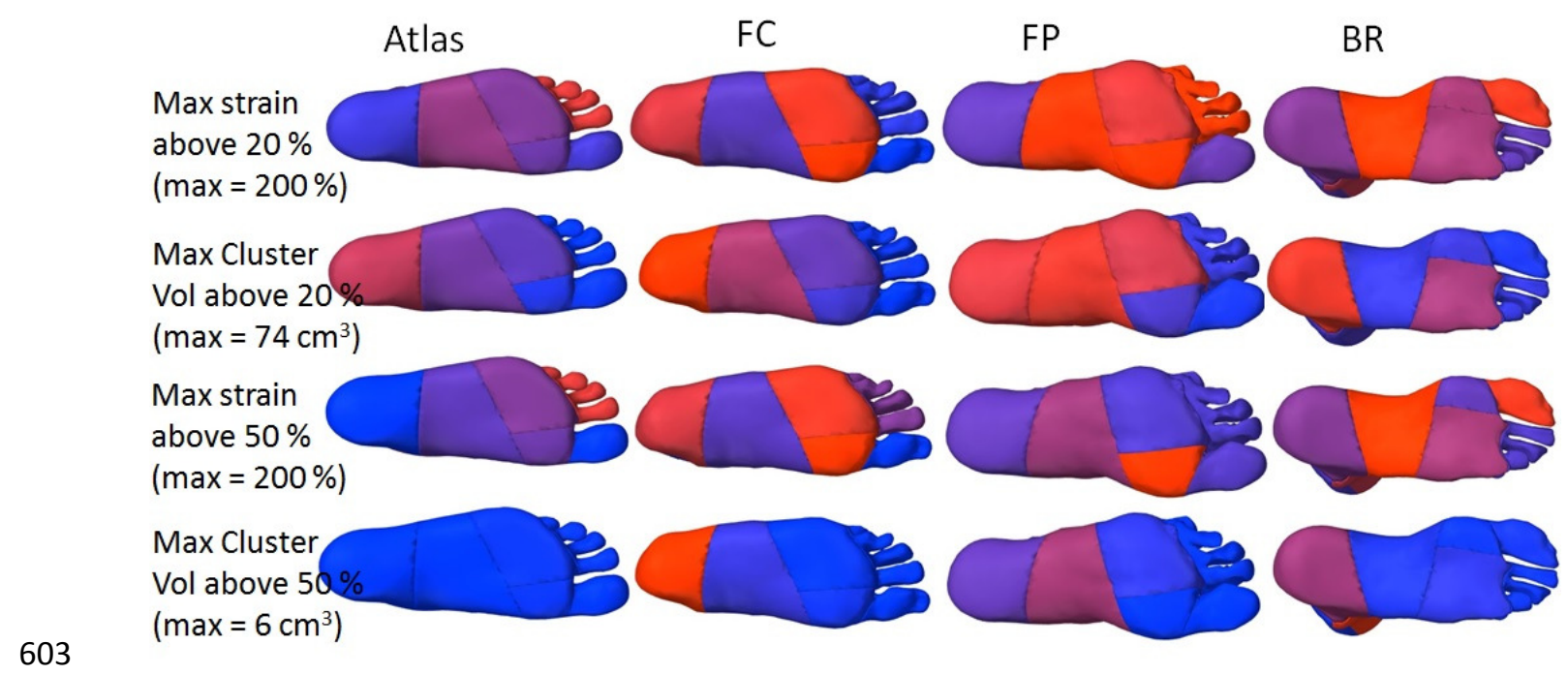

604

605 
Figure 6
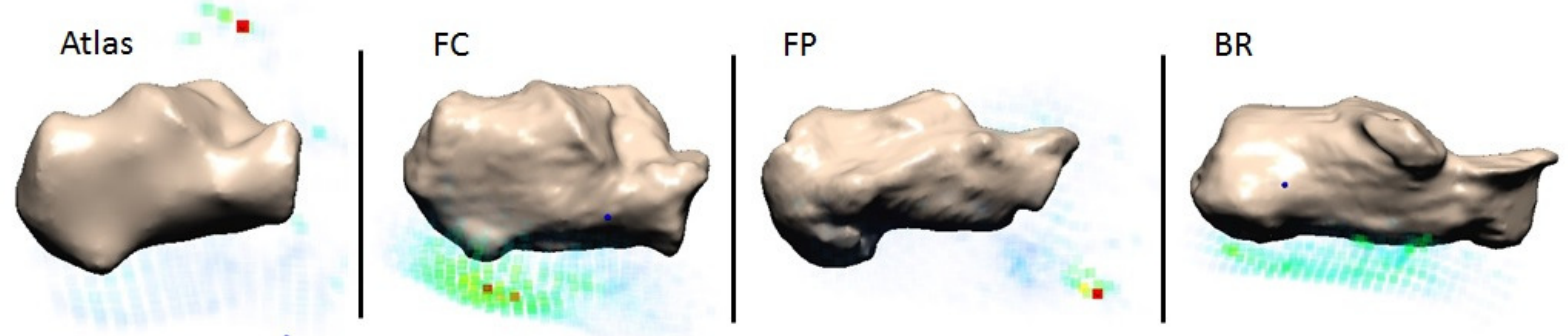

607

608

609 
Table 1

\begin{tabular}{|c|c|c|c|c|c|c|c|c|}
\hline Threshold 20\% & Atlas & & FC & & FP & & BR & \\
\hline & \begin{tabular}{|l|} 
max cluster \\
volume in \\
cm3
\end{tabular} & $\begin{array}{l}\max \text { VM } \\
\text { strain }\end{array}$ & \begin{tabular}{|l|} 
max cluster \\
volume in \\
$\mathrm{cm} 3$
\end{tabular} & \begin{tabular}{|l|} 
max VM \\
strain \\
\end{tabular} & \begin{tabular}{|l|} 
max cluster \\
volume in \\
cm3
\end{tabular} & \begin{tabular}{l|} 
max VM \\
strain
\end{tabular} & $\begin{array}{l}\text { max cluster } \\
\text { volume in } \\
\mathrm{cm} 3\end{array}$ & $\begin{array}{l}\text { max VM } \\
\text { strain }\end{array}$ \\
\hline (1) Achilles tendon & 7.3 & $85 \%$ & 0.5 & $27 \%$ & 5.7 & $90 \%$ & 17.9 & $69 \%$ \\
\hline (2) Top of the foot & 7.0 & $145 \%$ & 3.8 & $74 \%$ & 31.1 & $399 \%$ & 21.3 & $142 \%$ \\
\hline (3) Heel & 50.8 & $43 \%$ & 74.4 & $154 \%$ & 57.5 & $69 \%$ & 63.0 & $94 \%$ \\
\hline (4) Medio foot & 30.2 & $107 \%$ & 45.2 & $63 \%$ & 62.2 & $196 \%$ & 14.1 & $205 \%$ \\
\hline (5) 1st meta & 9.1 & $78 \%$ & 17.5 & $348 \%$ & 19.6 & $235 \%$ & 15.7 & $118 \%$ \\
\hline (6) 4 other meta & 24.3 & $91 \%$ & 26.1 & $174 \%$ & 54.5 & $159 \%$ & 42.6 & $117 \%$ \\
\hline (7) Hallux & 2.7 & $49 \%$ & 0.0 & $0 \%$ & 6.5 & $74 \%$ & 7.6 & $179 \%$ \\
\hline (8) 4 other toes & 0.7 & $161 \%$ & 2.7 & $31 \%$ & 14.2 & $326 \%$ & 14.0 & $69 \%$ \\
\hline Mean & 16.5 & $95 \%$ & 21.3 & $109 \%$ & 31.4 & $194 \%$ & 24.5 & $124 \%$ \\
\hline STD & 17.3 & 42ppt & 26.7 & $114 \mathrm{ppt}$ & 23.5 & $121 \mathrm{ppt}$ & 18.7 & 49ppt \\
\hline
\end{tabular}

611

26.7 114ppt

23.5 121ppt

612

613

614 
Table 2

\begin{tabular}{|c|c|c|c|c|c|c|c|c|}
\hline Threshold $50 \%$ & Atlas & & FC & & FP & & BR & \\
\hline & $\begin{array}{l}\text { max cluster } \\
\text { volume in } \\
\mathrm{cm} 3\end{array}$ & $\begin{array}{l}\text { max VM } \\
\text { strain }\end{array}$ & $\begin{array}{l}\text { max cluster } \\
\text { volume in } \\
\mathrm{cm} 3\end{array}$ & $\begin{array}{l}\text { max VM } \\
\text { strain }\end{array}$ & $\begin{array}{l}\text { max cluster } \\
\text { volume in } \\
\mathrm{cm} 3\end{array}$ & $\begin{array}{l}\text { max VM } \\
\text { strain }\end{array}$ & $\begin{array}{l}\text { max cluster } \\
\text { volume in } \\
\mathrm{cm} 3\end{array}$ & $\begin{array}{l}\text { max VM } \\
\text { strain }\end{array}$ \\
\hline (1) Achilles tendon & 0.1 & $85 \%$ & 0.0 & $0 \%$ & 0.3 & $90 \%$ & 0.5 & $55 \%$ \\
\hline (2) Top of the foot & 0.2 & $145 \%$ & 0.0 & $52 \%$ & 2.7 & $121 \%$ & 5.5 & $142 \%$ \\
\hline (3) Heel & 0.0 & $0 \%$ & 21.6 & $154 \%$ & 2.1 & $69 \%$ & 3.4 & $94 \%$ \\
\hline (4) Medio foot & 0.0 & $67 \%$ & 1.2 & $63 \%$ & 3.5 & $113 \%$ & 0.7 & $205 \%$ \\
\hline (5) 1st meta & 0.2 & $78 \%$ & 0.4 & $348 \%$ & 0.1 & $235 \%$ & 0.3 & $69 \%$ \\
\hline (6) 4 other meta & 0.2 & $91 \%$ & 0.1 & $174 \%$ & 1.0 & $56 \%$ & 1.0 & $117 \%$ \\
\hline (7) Hallux & 0.0 & $0 \%$ & 0.0 & $0 \%$ & 0.0 & $59 \%$ & 0.5 & $179 \%$ \\
\hline (8) 4 other toes & 0.1 & $161 \%$ & 0.0 & $92 \%$ & 0.1 & $53 \%$ & 0.3 & $57 \%$ \\
\hline Mean & 0.1 & $79 \%$ & 2.9 & $110 \%$ & 1.2 & $100 \%$ & 1.5 & $115 \%$ \\
\hline STD & 0.1 & 59ppt & 7.6 & $115 p p t$ & 1.4 & $61 \mathrm{ppt}$ & 1.9 & $57 \mathrm{ppt}$ \\
\hline
\end{tabular}

617 